\title{
Natural spring cell substitutes of simplex finite elements
}

\author{
IOANNIS DOLTSINIS \\ University of Stuttgart \\ Faculty of Aerospace Engineering and Geodesy \\ Pfaffenwaldring 27, 70569 Stuttgart \\ GERMANY
}

\begin{abstract}
Spring cell models are presented which derive from the natural description of simplex finite elements, that is in conformity with the geometry of the triangle in the plane and of the tetrahedron in space. Thereby, the spring cells are interpreted as part of the finite elements. The deduction of two spring cells as defective substitutes is demonstrated for the triangular element. One approximates the flexibility matrix of the element, the other approximates the stiffness matrix. The performance with respect to the finite element is analyzed, the issue of elastic anisotropy is discussed. In space, the spring cell substitute of the tetrahedral element is derived from the flexibility matrix, an inherent difference to the plane case is pointed out. Remarks on the implication of plasticity are added. Theaccountgivesabriefsummaryofrecentworkonthesubject.
\end{abstract}

Key-Words: Simplex finite elements, natural formalism, spring cells, elasticity, anisotropy, plasticity.

Received: September 27, 2019. Revised: February 27, 2020. Accepted: April 23, 2020. Published: April 29, 2020.

\section{Introduction}

Substitution regarding continuum structures and frameworks is noticed in the literature in both directions. For a representation of continuum structures by frameworks refer to the early work in [1]. Conversely, the handling of the mechanics of framework structures as for continua in [2]. While spring lattice methods and their applicability in modelling continuum structures in place of finite elements is still discussed [3], the present account just demonstrates a direct way to define spring cell substitutes of simplex finite elements in elasticity, investigates their performance when loaded and discusses the implication of plasticity.

The numerical modelling of deformable continua by spring lattices instead of finite elements is attracting interest by a number of arguments like simplified discrete representation, microstructural modelling [4], uniaxial material law, resolution of progressing damage and failure, ( Fig 1), and facilitated programming. Spring lattice methods refer to assemblies of bar elements covering the domain occupied by the continuum, (Fig 2). The attempted alternative suggests a comparison of the constituing spring cells with the continuum element. In this regard the performance of the simplest cell configurations, the triangular one in the plane and the tetrahedral in the three-dimensional space is investigated against the corresponding finite elements in constant strain.

Other than defining the spring cell a priori and
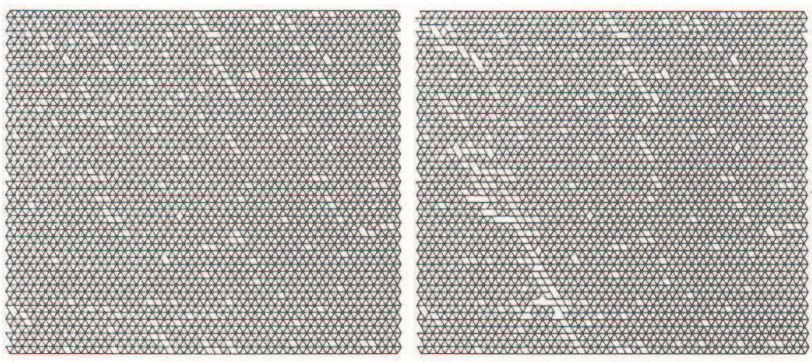

Figure 1: Evolution of damage in elastic spring lattice under shear [5]. Randomly distributed material strength.

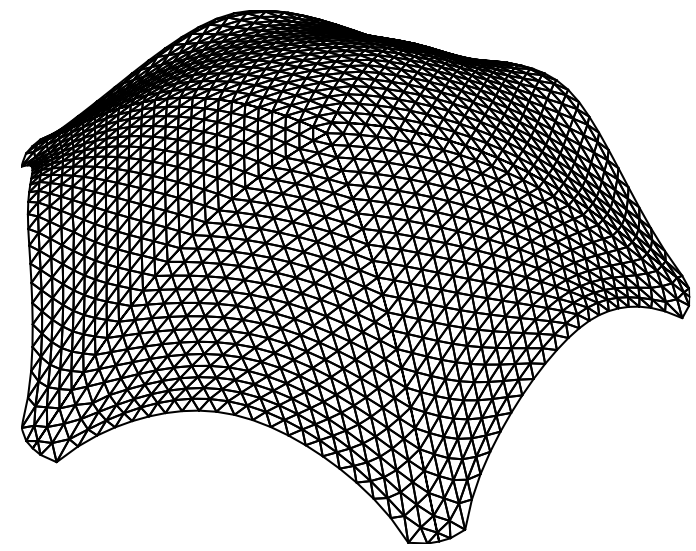

Figure 2: Spring lattice model representing membrane shell as a reticulated bar structure [6]. In a continuum approach the mesh would be covered by facet finite elements. 
attempting equivalence with the continuum element, the reported approach conceives the spring cell as part of the finite element. The concept takes advantage of the natural formalism introduced as early as in [7] such that the behaviour of the triangular element in the plane and of the tetrahedron in space is expressed in terms of quantities directed along the sides of the element and the edges respectively. The definition of two spring cell models is demonstrated for the triangular element: one covers in part the stiffness matrix of the element, the other analogously the flexibility matrix. In addition to isotropic elasticity the implication of anisotropy is commented. Both spring cell models are defective substitutes of the finite element and differ in their properties, which suggests comparison with respect to performance. The issue is investigated in dependence of the shape whereby the triangular spring cell models are contrasted with the finite element.

The spring cell in three-dimensional space is referred to the tetrahedral finite element. The diagonal entities of the flexibility matrix define the individual behaviour of the spring members of the cell. In contrast to the plane case of the triangle, there is no possibility to make the off-diagonal entities vanish such that complete substitution of the tetrahedral finite element is achieved. Beyond elasticity, plastic yielding in the spring cell necessitates caring for the individuality of the members, which is opposed to the unique behaviour of the continuum element.

The remainder of the text is organized as follows: Section 2 recalls the natural terminology for simplex finite elements, Section 3 presents two spring cell models as defective substitutes of the triangular finite element, Section 4 analyzes the performance of the two cell models in covering the finite element and Section 5 deals with elastic anisotropy. Section 6 is concerned with the spring cell in space referring to the tetrahedral element. Section 7 discusses the implication of plasticity in the spring cell context. Conclusions are summarized in Section 8.

\section{The natural formalism}

The natural formalism in finite element structural analysis refers to quantities that conform with the geometry of the element [7]. The constant strain elements, the four-node tetrahedron in space and the three-node triangle in the plane, (Fig 3), are of particular interest when discussing the introduction of spring cells because static and kinematic quantities are defined along the direction of the prospective springs. The lines connecting nodal points with lengths arranged as a diagonal matrix $\mathbf{l}=\left\lceil l_{\vartheta}\right\rfloor$ specify the natu-
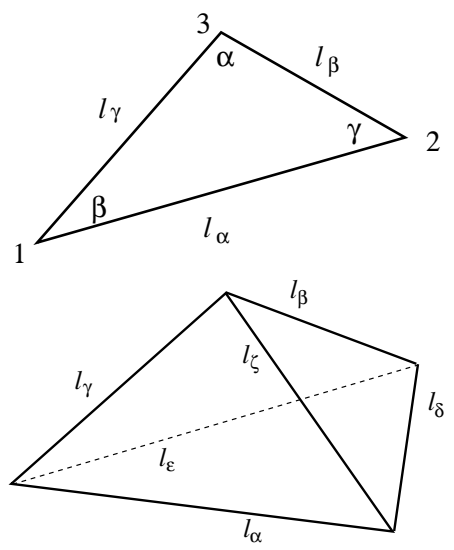

Figure 3: Triangle and tetrahedron. Connecting lines with lengths $l_{\vartheta}$ define the natural directions.

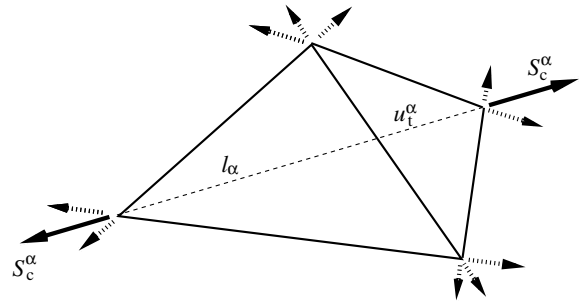

Figure 4: Forces $\mathbf{S}_{\mathrm{c}}$ imposed along the edges of the tetrahedron induce elongations $\mathbf{u}_{\mathrm{t}}$.

ral directions, quantities defined along the natural directions are denoted either total or component. The elongations of lines connecting nodes define the total displacements collected in the vector array $\mathbf{u}_{\mathrm{t}}=$ $\left\{\mathbf{u}_{\mathrm{t}}^{\vartheta}\right\}$, (Fig 4), the unit elongations are the total strains in $\varepsilon_{\mathrm{t}}=\left\{\varepsilon_{\mathrm{t}}^{\vartheta}\right\}$.

Regarding statics, the forces acting at the nodal points are composed of constituents along the natural directions, (Fig 4), collected in the vector array $\mathbf{S}_{\mathrm{c}}=\left\{S_{\mathrm{c}}^{\vartheta}\right\}$. These are the resultants of the component stresses in $\sigma_{\mathrm{c}}=\left\{\sigma_{\mathrm{c}}^{\vartheta}\right\}$ and satisfy in the element of volume $V$ the work equivalence

$$
V \varepsilon_{\mathrm{t}}^{\mathrm{t}} \boldsymbol{\sigma}_{\mathrm{c}}=\mathbf{u}_{\mathrm{t}}^{\mathrm{t}} \mathbf{S}_{\mathrm{c}} .
$$

In linear elasticity the relationship between the forces $\mathbf{S}_{\mathrm{c}}$ and the elongations $\mathbf{u}_{\mathrm{t}}$ is obtained as

$$
\mathbf{u}_{\mathrm{t}}=\frac{1}{V} \mathbf{l} \phi_{\mathrm{N}} \mathbf{l} \mathbf{S}_{\mathrm{c}}=\mathbf{f}_{\mathrm{N}} \mathbf{S}_{\mathrm{c}},
$$

where $\mathbf{f}_{\mathrm{N}}$ denotes the flexibility matrix of the element. The flexibility matrix of the elastic material, $\phi_{\mathrm{N}}$, computes the strain $\varepsilon_{\mathrm{t}}$ along the sides for the stress $\sigma_{\mathrm{c}}$ induced by the imposed forces:

$$
\varepsilon_{\mathrm{t}}=\mathbf{l}^{-1} \mathbf{u}_{\mathrm{t}}=\phi_{\mathrm{N}}\left(\frac{1}{V} \mathbf{l S}_{\mathrm{c}}\right)=\phi_{\mathrm{N}} \boldsymbol{\sigma}_{\mathrm{c}}
$$



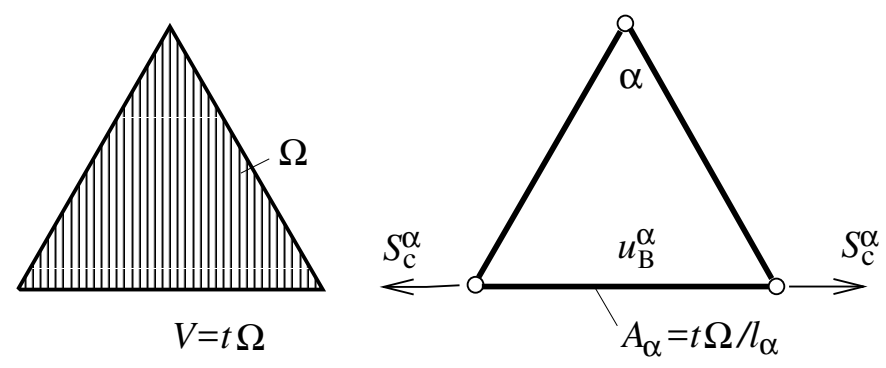

Figure 5: Solid triangle (area $\Omega$, thickness $t$ ), left. Three-bar assembly (bar-length $l$, cross-section area $A)$, right.

The symbolism applies to the two- and the threedimensional case with the content adapted.

\section{Two triangular spring cells}

The natural representation of the simplex triangular finite element -with reference to the directions along the sides- proves useful in deducing two variants of the triangular spring cell: one approximating the flexibility matrix of the element, the other the stiffness matrix [8]. The volume of the triangle with area $\Omega$ and unique thickness $t$ is $V=\Omega t$, the flexibility matrix of the elastic material in plane stress with elasticity parameters $E, \nu$ is

$$
\begin{gathered}
\frac{1}{E}\left[\begin{array}{ccc}
\phi_{\mathrm{N}}= \\
\cos \gamma-\nu \sin ^{2} \gamma & \cos ^{2} \beta-\nu \sin ^{2} \beta \\
\operatorname{sym} & & \cos ^{2} \alpha-\nu \sin ^{2} \alpha \\
1 &
\end{array}\right] \\
=\frac{1}{E}\left[\begin{array}{ccc}
1 & c & b \\
& 1 & a \\
\operatorname{sym} & & 1
\end{array}\right]
\end{gathered}
$$

The abbreviations

$$
\begin{aligned}
& c=\left(\cos ^{2} \gamma-\nu \sin ^{2} \gamma\right), \\
& b=\left(\cos ^{2} \beta-\nu \sin ^{2} \beta\right), \\
& a=\left(\cos ^{2} \alpha-\nu \sin ^{2} \alpha\right),
\end{aligned}
$$

help shorten subsequent expressions.

The two spring cell models derive from the natural finite element as follows.

\subsection{The flexibility cell}

Use of eqn (2) with just the diagonal of the material flexibility matrix, eqn (4), leads to the flexibility version of the spring cell governed by

$$
\mathbf{u}_{\mathrm{B}}={ }_{E}^{\overline{1}} 1 \mathbf{A}^{-1} \mathbf{S}_{\mathrm{c}}=\mathbf{f}_{\mathrm{B}} \mathbf{S}_{\mathrm{c}}
$$

where $f_{B}$ denotes the diagonal flexibility matrix of the spring cell. Equation (6) computes the elongations $\mathbf{u}_{\mathrm{B}}$ for forces $\mathbf{S}_{\mathrm{c}}$ imposed on a skeletal triangle built of pin-joined bars, (Fig 5), with cross-section areas

$$
\mathbf{A}=\Omega t \mathbf{l}^{-1} \text {. }
$$

The structure of the material flexibility matrix in eqn (4) reveals the spring cell as the part of the finite element left after removal of the geometrical and physical coupling quantified by the off-diagonal entities of the matrix.

\subsection{The stiffness cell}

The alternative stiffness approach to the spring cell refers to the inverse of eqn (2)

$$
\mathbf{S}_{\mathrm{c}}=\Omega t \mathbf{l}^{-1} \boldsymbol{\phi}_{\mathrm{N}}^{-1} \mathbf{l}^{-1} \mathbf{u}_{\mathrm{t}}=\mathbf{k}_{\mathrm{N}} \mathbf{u}_{\mathrm{t}},
$$

where $\mathbf{k}_{\mathrm{N}}$ denotes the stiffness matrix of the elastic finite element. The inverse of the material flexibility matrix is denoted $\boldsymbol{\kappa}_{\mathrm{N}}=\phi_{\mathrm{N}}^{-1}$. Use in eqn (8) of its diagonal

$\boldsymbol{\kappa}_{\mathrm{D}}=\frac{E}{\operatorname{Det}}\left[\begin{array}{ccc}1-a^{2} & & \\ & 1-b^{2} & \\ & & 1-c^{2}\end{array}\right]=E \boldsymbol{\Delta}$,

with the determinant

$$
\text { Det }=\left|\mathrm{E} \boldsymbol{\phi}_{\mathrm{N}}\right|=1-\left(\mathrm{a}^{2}+\mathrm{b}^{2}+\mathrm{c}^{2}\right)+2 \mathrm{abc},
$$

gives

$$
\mathbf{S}_{\mathrm{B}}=E \mathbf{A}_{\mathrm{k}} \mathbf{l}^{-1} \mathbf{u}_{\mathrm{t}}=\mathbf{k}_{\mathrm{B}} \mathbf{u}_{\mathrm{t}} .
$$

The diagonal matrix $\mathbf{k}_{\mathrm{B}}$ defines the stiffness of the spring cell. Equation (11) computes the stress resultants $\mathbf{S}_{\mathrm{B}}$ as for elongations $\mathbf{u}_{\mathrm{t}}$ imposed on the pinjoined bars of a skeletal triangle with cross-section areas

$$
\mathbf{A}_{\mathrm{k}}=\Delta \mathbf{A}
$$

and $\mathbf{A}$ comprises the cross section areas of the previous flexibility approach in eqn (7).

\section{Analysis of performance}

The described spring cell models offer two distinctly defective substitutes for the triangular finite element. The stiffness and the flexibility approach coincide only in the ideal case of perfect equivalence with the finite element which requires the triangle to be regular $(\alpha=\beta=\gamma)$ and to be of a material with the lateral contraction coefficient $\nu=1 / 3$. For such a constellation of geometrical shape and material parameter the off-diagonal entities in the material flexibility matrix, 
eqn (4), vanish and so in its inverse. The ideal configuration is not necessarily met in computations, which rather favour the flexibility variant [3], [9]. This puts importance on the performance analysis of the two spring cell models in dependence of a varying shape [10].

Contrasting eqn (6) with eqn (11), the flexibility and the stiffness cell model differ by the calculatory cross-section area of the constituing bar members, and so the stiffness matrices $\mathbf{k}_{\mathrm{B}}, \mathbf{f}_{\mathrm{B}}^{-1}$. Eventually eqn (12) relates the two models by

$$
\mathbf{k}_{\mathrm{B}} \mathbf{f}_{\mathrm{B}}=\mathbf{A}_{\mathrm{k}} \mathbf{A}^{-1}=\boldsymbol{\Delta},
$$

where $\Delta$ is the diagonal matrix appearing in eqn (9).

Equation (13) is useful in relating stiffness and flexibility of the two cell models; it does not inform on the approximation of the continuum finite element. The latter issue will be explored by means of the elastic energy stored in the different discretization units under imposed forces $\mathbf{S}_{\mathrm{c}}$. It should be kept in mind, that the cell models under study are based on simplifications of the material flexibility matrix and of its inverse. On account of eqn (2) the elastic energy of the finite element under force is

$$
W_{\mathrm{N}}=\frac{1}{2} \mathbf{S}_{\mathrm{c}}^{\mathrm{t}} \mathbf{f}_{\mathrm{N}} \mathbf{S}_{\mathrm{c}}=\frac{\Omega t}{2} \boldsymbol{\sigma}_{\mathrm{c}}^{\mathrm{t}} \boldsymbol{\phi}_{\mathrm{N}} \boldsymbol{\sigma}_{\mathrm{c}} .
$$

The transition to the stress $\sigma_{\mathrm{c}}$ observes the work equivalence of eqn (1). Standardization of the energy by the element volume, the modulus of elasticity and the magnitude of the imposed stress defines a test quantity for the comparison of units of equal volume $\Omega t$ and elasticity parameters:

$$
\bar{w}_{\mathrm{N}}=\frac{2 E}{\Omega t} \frac{W_{\mathrm{N}}}{\boldsymbol{\sigma}_{\mathrm{c}}^{\mathrm{t}} \boldsymbol{\sigma}_{\mathrm{c}}}=\frac{\boldsymbol{\sigma}_{\mathrm{c}}^{\mathrm{t}}\left(E \phi_{\mathrm{N}}\right) \boldsymbol{\sigma}_{\mathrm{c}}}{\boldsymbol{\sigma}_{\mathrm{c}}^{\mathrm{t}} \boldsymbol{\sigma}_{\mathrm{c}}} .
$$

The last expression in eqn (15) reveals the test quantity as the Rayleigh quotient bounded by the eigenvalues $\lambda_{\mathrm{N}}$ of the matrix $E \phi_{\mathrm{N}}$ :

$$
\left(\lambda_{\mathrm{N}}\right)_{\min } \leq \bar{w}_{\mathrm{N}} \leq\left(\lambda_{\mathrm{N}}\right)_{\max } .
$$

The energy bounds are depicted in Fig 6 for a material with the coefficient of lateral contraction $\nu=1 / 3$. The configuration of the triangle enters via the three angles $\alpha, \beta$ and $\gamma=180^{\circ}-(\alpha+\beta)$.

The flexibility approximation computes the bar elongations by eqn (6) which on account of eqn (7) gives the elastic energy as

$$
W_{\mathrm{f}}=\frac{1}{2} \mathbf{S}_{\mathrm{c}}^{\mathrm{t}} \mathbf{f}_{\mathrm{B}} \mathbf{S}_{\mathrm{c}}=\frac{\Omega t}{2 E} \boldsymbol{\sigma}_{\mathrm{c}}^{\mathrm{t}} \mathbf{I} \boldsymbol{\sigma}_{\mathrm{c}},
$$

where I denotes the identity operator. The test quantity is here fixed to unity

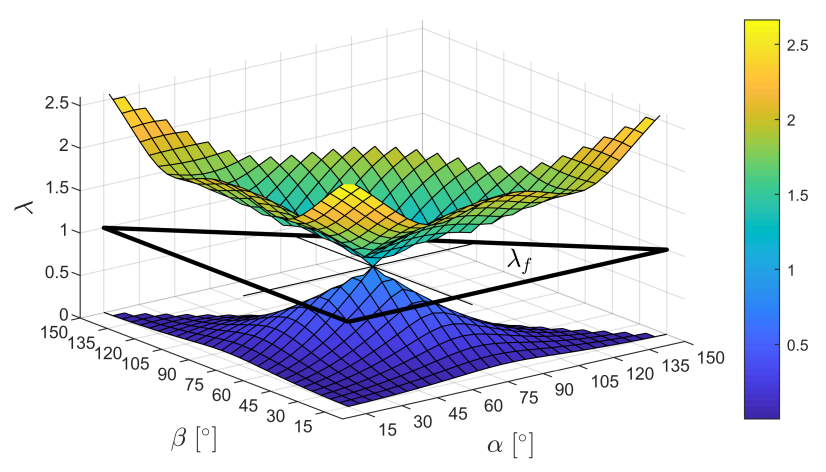

Figure 6: Bounds of the energy $\left(\lambda_{\mathrm{N}}\right)_{\min } \leq \bar{w}_{\mathrm{N}} \leq$ $\left(\lambda_{\mathrm{N}}\right)_{\max }$ for the triangular element as a function of the angles $(\nu=1 / 3)$. The plane at level one marks the flexibility cell $\left(\lambda_{\mathrm{f}}=1\right)$. Coincidence for the regular triangle.

$$
\bar{w}_{\mathrm{f}}=\frac{2 E}{\Omega t} \frac{W_{\mathrm{f}}}{\boldsymbol{\sigma}_{\mathrm{c}}^{\mathrm{t}} \boldsymbol{\sigma}_{\mathrm{c}}}=\frac{\boldsymbol{\sigma}_{\mathrm{c}}^{\mathrm{t}} \mathbf{I} \boldsymbol{\sigma}_{\mathrm{c}}}{\boldsymbol{\sigma}_{\mathrm{c}}^{\mathrm{t}} \boldsymbol{\sigma}_{\mathrm{c}}}=\lambda_{\mathrm{f}}=1 .
$$

This marks a level which facilitates visual comparison in the graphics.

The elastic energy for the stiffness approximation is obtained with the bar elongations from eqn (11) using eqn (13) for the inverse cell stiffness

$$
W_{\mathrm{k}}=\frac{1}{2} \mathbf{S}_{\mathrm{c}}^{\mathrm{t}}\left(\mathbf{f}_{\mathrm{B}} \boldsymbol{\Delta}^{-1}\right) \mathbf{S}_{\mathrm{c}}=\frac{\Omega t}{2 E} \boldsymbol{\sigma}_{\mathrm{c}}^{\mathrm{t}} \boldsymbol{\Delta}^{-1} \boldsymbol{\sigma}_{\mathrm{c}}
$$

The test quantity is built with the diagonal matrix $\boldsymbol{\Delta}^{-1}=\left\lceil\Delta_{\vartheta}\right\rfloor^{-1}, \vartheta=\alpha, \beta, \gamma$,

$$
\bar{w}_{\mathrm{k}}=\frac{2 E}{\Omega t} \frac{W_{\mathrm{k}}}{\boldsymbol{\sigma}_{\mathrm{c}}^{\mathrm{t}} \boldsymbol{\sigma}_{\mathrm{c}}}=\frac{\boldsymbol{\sigma}_{\mathrm{c}}^{\mathrm{t}} \boldsymbol{\Delta}^{-1} \boldsymbol{\sigma}_{\mathrm{c}}}{\boldsymbol{\sigma}_{\mathrm{c}}^{\mathrm{t}} \boldsymbol{\sigma}_{\mathrm{c}}} .
$$

It is bounded by the eigenvalues $\lambda_{\mathrm{k}}=\Delta_{\vartheta}^{-1}$ as follows

$$
\begin{array}{r}
\left(\lambda_{\mathrm{k}}\right)_{\min }=\left(\Delta_{\vartheta}^{-1}\right)_{\min } \leq \bar{w}_{\mathrm{k}} \\
\leq\left(\Delta_{\vartheta}^{-1}\right)_{\max }=\left(\lambda_{\mathrm{k}}\right)_{\max }, \quad \vartheta=\alpha, \beta, \gamma .
\end{array}
$$

The domain of variation of the energy $\bar{w}_{\mathrm{k}}$ for the triangular stiffness cell is displayed in Fig 7 as a function of the angles. In the figure the plane at level one pertains to the flexibility cell, $\lambda_{\mathrm{f}}=1$, eqn (18).

The investigations are seen to favour the flexibility cell in the large, but in certain constellations the stiffness cell approximation is found closer to the finite element. The latter cell behaves stiffer than its flexibility counterpart.

\section{Plane anisotropy}

In case that the material constants are not in common but differ along the natural directions [6], anisotropy 


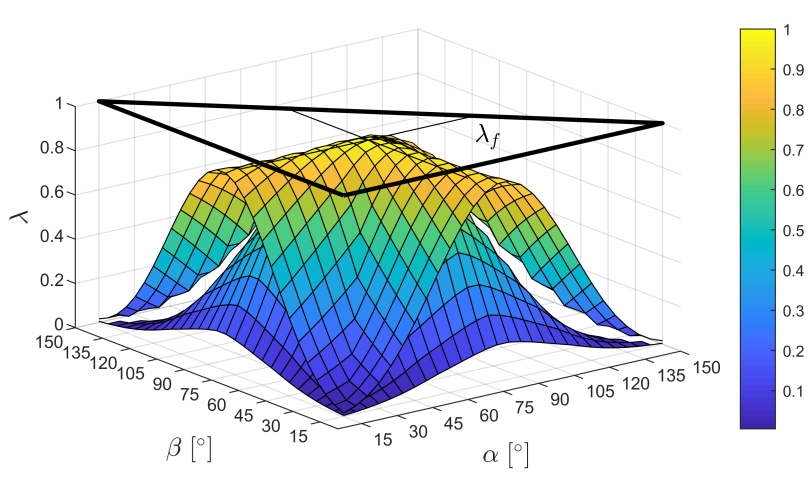

Figure 7: Bounds of the energy $\left(\lambda_{\mathrm{k}}\right)_{\min } \leq \bar{w}_{\mathrm{k}} \leq$ $\left(\lambda_{\mathrm{k}}\right)_{\max }$ for the triangular stiffness cell as a function of the angles $(\nu=1 / 3)$. The plane at level one marks the flexibility cell $\left(\lambda_{\mathrm{f}}=1\right)$. Coincidence for the regular triangle.

alters the material flexibility matrix of eqn (4) to

$$
\begin{gathered}
\phi_{\mathrm{NA}}= \\
{\left[\begin{array}{ccc}
\frac{1}{E_{\alpha}} & \frac{\cos ^{2} \gamma-\nu_{\beta} \sin ^{2} \gamma}{E_{\beta}} & \frac{\cos ^{2} \beta-\nu_{\gamma} \sin ^{2} \beta}{E_{\gamma}} \\
\frac{\cos ^{2} \gamma-\nu_{\alpha} \sin ^{2} \gamma}{E_{\alpha}} & \frac{1}{E_{\beta}} & \frac{\cos ^{2} \alpha-\nu_{\gamma} \sin ^{2} \alpha}{E_{\gamma}} \\
\frac{\cos ^{2} \beta-\nu_{\alpha} \sin ^{2} \beta}{E_{\alpha}} & \frac{\cos ^{2} \alpha-\nu_{\beta} \sin ^{2} \alpha}{E_{\beta}} & \frac{1}{E_{\gamma}}
\end{array}\right]}
\end{gathered}
$$

Symmetry imposes relationships between the offdiagonal entities. Focusing on elastic orthotropy in plane stress, the material properties enter the description with the parameters $E_{1}, \nu_{1}$ and $E_{2}, \nu_{2}$ along the orthotropy directions and with the parameter $G$ defined as a physical quantity on its own. The symmetry requirement in the orthotropic system implies that

$$
\frac{\nu_{2}}{E_{2}}=\frac{\nu_{1}}{E_{1}}=\left\langle\frac{\nu}{E}\right\rangle=\frac{1}{2}\left(\frac{\nu_{1}}{E_{1}}+\frac{\nu_{2}}{E_{2}}\right) .
$$

The introduction of the common quotient facilitates the formalism which is transformed to the natural reference system [6]. Substitution of the constant strain triangle by a pin-joined three-bar truss requires the three off-diagonal entities of the ensuing flexibility matrix to vanish. Taking the orthotropic $E_{1}, \nu_{1}$ along the side opposite to the angle $\alpha$, (Fig 8), the requirement relates the other two angles to $\nu_{1}$ :

$$
\tan ^{-2} \beta=\tan ^{-2} \gamma=\nu_{1}
$$

The third vanishing condition specifies $\nu_{1}$ from

$$
\frac{\nu_{1}}{E_{1}}=\sqrt{\frac{1}{4 G^{2}}+\frac{1}{E_{1} E_{2}}}-\frac{1}{2 G}=\frac{\nu_{2}}{E_{2}},
$$

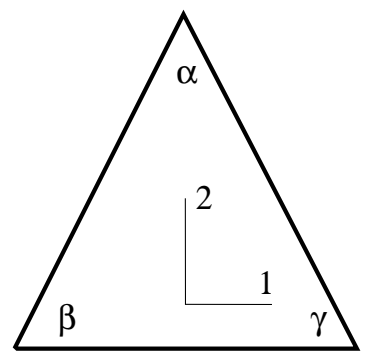

Figure 8: Triangular element of orthotropic material.

and determines by virtue of eqn (24) the geometry of the isosceles triangle capable of substitution by pinjoined bars along the sides. In case of isotropy, $E_{2}=$ $E_{1}=E, \nu_{2}=\nu_{1}=\nu, 2 G=E /(1+\nu)$, eqn (25) is satisfied for $\nu=1 / 3$, the triangle then being a regular one as stated previously.

\section{Spring cell in space - the tetrahe- dron}

With reference to Section 2 the relationship between the elongations in $\mathbf{u}_{\mathrm{t}}$ and the imposed forces in $\mathbf{S}_{\mathrm{c}}$ for the tetrahedral finite element at constant strain is defined by eqn (2), the matrix arrays appropriately adjusted [6]. The natural flexibility matrix for the elastic material conforms with the tetrahedron

$$
\phi_{\mathrm{N}}=\frac{1}{E}\left[\begin{array}{ccccc}
1 & \phi_{\alpha \beta} & \phi_{\alpha \gamma} & \cdots & \phi_{\alpha \zeta} \\
& 1 & \phi_{\beta \gamma} & \cdots & \phi_{\beta \zeta} \\
& & 1 & \cdots & \vdots \\
& & & \ddots & \vdots \\
\text { sym } & & & & 1
\end{array}\right] \text {, }
$$

and the entities of the matrix are

$$
\begin{array}{r}
\phi_{\vartheta \psi}=\cos ^{2}(\vartheta, \psi)-\nu \sin ^{2}(\vartheta, \psi), \\
\vartheta, \psi=\alpha, \beta, \cdots, \zeta .
\end{array}
$$

This defines in conjunction with eqn (2) the flexibility matrix $\mathbf{f}_{\mathrm{N}}$ of the tetrahedral element. The diagonal $\frac{1}{E} \mathbf{I}$ of $\phi_{\mathrm{N}}$ enters the flexibility matrix of the spring cell

$$
\mathbf{f}_{\mathrm{B}}=\frac{1}{V E} \mathbf{l}^{2}=\frac{1}{E} \mathbf{l A}^{-1},
$$

where $V$ is the volume of the tetrahedron and $\mathbf{A}$ comprises the calculatory cross-section areas computed for the bars with lengths in $\mathrm{l}$ :

$$
\mathbf{A}=\left\lceil A_{\vartheta}=\frac{V}{l_{\vartheta}}\right\rceil=V \mathbf{l}^{-1}, \quad \vartheta=\alpha, \beta, \cdots, \zeta .
$$



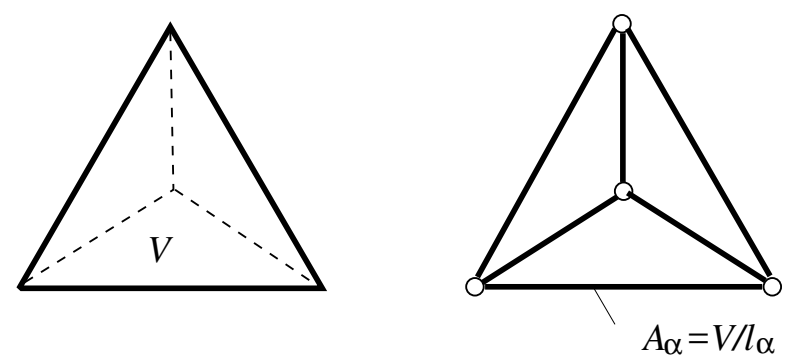

Figure 9: Solid tetrahedron (volume $V$ ), left. Six-bar assembly (bar-length $l$, cross-section area $A$ ), right.

Thereby the geometrical data in eqn (2), adjusted to the tetrahedron, are assigned to a skeletal element assembled of elastic bar members, the springs, connecting the nodes, (Fig 9).

The spring cell assembled of individually acting bar members does not substitute the continuum element completely unless the off-diagonal entities in eqn (26) vanish. This is the case where the angular distance $(\vartheta, \psi)$ between edges satisfies the condition

$\cos ^{2}(\vartheta . \psi)-\nu \sin ^{2}(\vartheta, \psi)=0, \quad \vartheta, \psi=\alpha, \beta, \cdots, \zeta$,

which is not everywhere possible in the tetrahedron. No satisfaction makes the cell defective with respect to element substitution to the degree that coupling terms are ignored.

For instance, the regular tetrahedron of a material with

$$
\nu=\tan ^{-2} 60^{\circ}=\frac{1}{3},
$$

fulfills the requirement for the four edges adjacent to the one considered, (Fig 9), while the opposed edge prohibits completion. The associated off-diagonal term is

$$
\cos ^{2} 90^{\circ}-\nu \sin ^{2} 90^{\circ}=-\frac{1}{3} .
$$

By consideration in eqn (26), an assembly of individually acting bars still discards with respect to the solid tetrahedral element the excess quantity

$$
\phi_{\mathrm{N}}-\frac{1}{E} \mathbf{I}=\frac{1}{E}\left[\begin{array}{cccccc}
0 & -\frac{1}{3} & 0 & 0 & 0 & 0 \\
& 0 & 0 & 0 & 0 & 0 \\
& & 0 & -\frac{1}{3} & 0 & 0 \\
& & & 0 & 0 & 0 \\
\text { sym } & & & & 0 & -\frac{1}{3} \\
& & & & & 0
\end{array}\right],
$$

which could be accounted for by interactions between the bar members. The nonzero entries in the matrix refer to opposed edges (see Fig 3).

\section{Remarks on plasticity}

The implementation of plasticity in the cell demands attention to the yield condition and to the plastic flow [3]. For the sake of brevity the essential formalism is displayed for the triangular element [11]. Regarding the stress state, the hydrostatic part is obtained with the imposed stress $\sigma_{\mathrm{c}}$ as

$$
\sigma_{\mathrm{H}}=\frac{1}{3} \mathbf{e}^{\mathrm{t}} \boldsymbol{\sigma}_{\mathrm{c}}, \quad \mathbf{e}=\{111\} .
$$

The equivalent stress $\bar{\sigma}$, the yield criterion, is computed from

$$
\begin{aligned}
\bar{\sigma}^{2} & =\frac{3}{2}\left[\boldsymbol{\sigma}_{\mathrm{c}}^{\mathrm{t}}(\mathbf{I}+\mathcal{C}) \boldsymbol{\sigma}_{\mathrm{c}}-3 \sigma_{\mathrm{H}}^{2}\right]= \\
& =\boldsymbol{\sigma}_{\mathrm{c}}^{\mathrm{t}} \boldsymbol{\sigma}_{\mathrm{c}}+\boldsymbol{\sigma}_{\mathrm{c}}^{\mathrm{t}}\left(\mathcal{C}-\frac{1}{2} \mathcal{B}\right) \boldsymbol{\sigma}_{\mathrm{c}}
\end{aligned}
$$

where the off-diagonal matrices

$$
\begin{gathered}
\mathcal{C}=\left[\begin{array}{ccc}
0 & \cos ^{2} \gamma & \cos ^{2} \beta \\
& 0 & \cos ^{2} \alpha \\
\operatorname{sym} & & 0
\end{array}\right], \\
\mathcal{B}=\left[\begin{array}{ccc}
0 & \sin ^{2} \gamma & \sin ^{2} \beta \\
& 0 & \sin ^{2} \alpha \\
\operatorname{sym} & & 0
\end{array}\right],
\end{gathered}
$$

import the trigonometry of the triangle.

The above helps assessing the yield point of the spring cell with respect to the triangular finite element. The first term in the last expression in eqn (33) is covered by the sum over the bar stresses in the cell. The second term, not appearing in the cell, accounts for the combined action of the imposed component stresses. Single entities vanish for

$$
\tan ^{2} \vartheta=2, \quad \vartheta=54.7356^{\circ},
$$

which is near regularity.

If the cell is forced to yield at once, the quantity $\boldsymbol{\sigma}_{\mathrm{c}}^{\mathrm{t}} \boldsymbol{\sigma}_{\mathrm{c}}$ provides a collective criterion as an approximation to the continuum one. Equivalence suggests compensation by an adjustment of the material yield stress. If the yield point is tested individually for each bar member, only certain geometric and stressing conditions will induce the collective yielding of the cell.

In general the yield point at a given stress state will differ between bars. A comparison with the plastic evolution in the continuum is meaningful if the cell is forced to yield at once upon satisfaction of the collective criterion. The plastic flow in the continuum element is stated in the form

$$
\mathrm{d} \boldsymbol{\eta}_{\mathrm{t}}=\frac{\mathrm{d} \bar{\eta}}{\bar{\sigma}} \boldsymbol{\sigma}_{\mathrm{c}}+\frac{\mathrm{d} \bar{\eta}}{\bar{\sigma}}\left(\mathcal{C}-\frac{1}{2} \mathcal{B}\right) \boldsymbol{\sigma}_{\mathrm{c}}
$$


where $\bar{\eta}$ denotes the accumulated equivalent plastic strain. The plastic flow in the bars corresponds to the first term in eqn (37), the additive term quantifies the disagreement between the cell and the finite element as discussed in connection with the equivalent stress.

It is noted that the matrix operator $(\mathcal{C}-0.5 \mathcal{B})$ in eqn (37) and eqn (33) becomes singular if the triangle satisfies $\tan ^{2} \vartheta=2$ in one of the angles. In the associated eigenvector the opposed bar member appears stress free as in the eigenvector

$$
\left\{0-\left(\cos ^{2} \beta-0.5 \sin ^{2} \beta\right)\left(\cos ^{2} \gamma-0.5 \sin ^{2} \gamma\right)\right\}
$$

associated with $\tan ^{2} \alpha=2$. Under such conditions of geometry and loading the finite element additive to plastic flow vanishes as does also in the collective yield criterion.

\section{Conclusion}

The natural approach originally suiting finite elements has been found useful in discussing the transition to the skeletal spring cell. The formalism is seen to offer a transparent access to the spring cell issue in elasticity. The deficiency of cells built as simple bar assemblies becomes immediately evident, the impact of imperfect configurations clear.

The exposed method addresses the degradation of the finite element to an assembly of bar members that constitute the spring cell. Two spring cell variants are feasible with reference to the natural formalism of the finite element which employs quantities along the sides of the triangle. The flexibility cell works with the diagonal entities of the finite element flexibility matrix, the stiffness cell analogously with the diagonal entities of the finite element stiffness matrix. The flexibility approach serves smoothly the purpose, and helps to deduce the cell alternatively on the stiffness basis. The approach is demonstrated for triangular units. As a consequence of the calculatory cross-section areas assigned to the bar members by either approximation the cell properties differ excepting the ideal case of the complete equivalence to the simplex finite element. The spring cell issue has been extended to plane anisotropic elasticity with orthotropy and isotropy as special cases.

Computations apparently suggest a preference for the flexibility model which motivates comparison of the two spring cell models with respect to their capability to substitute the triangular finite element in constant strain. The test quantity defined for the comparison is the Rayleigh quotient involving the specific elastic energy stored in the units under stress. The bounding eigenvalues pertaining to the individual units indicate the flexibility cell largely to approach the finite element closer than the stiffness cell but not always. Therefore a general superiority can not be claimed for the flexibility cell other than its inherent insensitivity to the shape of the triangle. The stiffness cell turns out to behave stiffer than its flexibility counterpart.

In three-dimensional elasticity, the definition of the spring cell in space is demonstrated with reference to the flexibility matrix of the simplex tetrahedral finite element. The spring cell may be interpreted as part of the element, the deficiency is pointed out. The tetrahedral finite element can not be substituted completely by the respective spring cell, while the triangular element can it under certain conditions of shape and material, a well established fact in the literature.

The implementation of plasticity in the cell is facilitated due to the uni-dimensional bar members but requires attention when modelling the continuum. Both, the yield criterion and the plastic flow are seen to meet with the finite element only under certain conditions of geometry and stressing. Otherwise, equivalence implies compensation of the defective representation which can be attempted by an adjustment of the material yield stress in the computation.

\section{References:}

[1] A. Hrennikoff, Solution of problems of elasticity by the frame-work-method, ASME J. Appl. Mech. 8, 1941, pp. 169-175.

[2] D. T. Wright, Membrane forces and buckling in reticulated shells, Proc. ASCE Struct. Div. 91, 1965, pp. 173-201.

[3] I. Doltsinis, M. Reck and V. Dias da Silva, Spring lattice models in the nonlinear analysis of membrane shells - an applicability study in elasticity, plasticity and damage, Comp. \& Struct. 10.1016/j.compstruc., 2019, 106174.

[4] M. Ostoja-Starzewski, Lattice models in micromechanics, Appl. Mech. Rev. 55, 2002, pp. 35-60.

[5] I. Doltsinis and R. Dattke, Numerical experiments on the rupture of brittle solids-variation of microstructure, loading and dimensions Int. $J$. Sol. Struct. 42, 2005, pp. 565-79.

[6] I. Doltsinis, From the natural continuum to the elastic spring cell in three and two dimensions - a flexibility approach, Eng. Computat. 10.1108/EC-11-2018-0543.

[7] J. Argyris, Three-dimensional anisotropic and inhomogeneous elastic media - matrix analysis for small and large displacements, IngenieurArchiv 34, 1965, pp. 33-55. 
[8] I. Doltsinis, A note on the flexibility and stiffness natural approach to the triangular spring cell, Eng. Computat. 35, 2018, pp. 130-139.

[9] T. Sá Marques, V. Dias da Silva and E. N. B. S. Júlio, Form finding of continua shells with lattice spring models Eng. Struct. 10.1016/j.engstruct.2019.109683

[10] I. Doltsinis, Stiffness vs. flexibility based triangular spring cell - study on performance $I S D$ University of Stuttgart Report, 2019.

[11] J. H. Argyris, D. W. Scharpf and J. B. Spooner, Die elastoplastische Berechnung von allgemeinen Tragwerken und Kontinua, IngenieurArchiv 37, 1969, pp. 326-52. 